Pacific Journal of Mathematics

ADMISSIBLE MODULES AND A CHARACTERIZATION O

GüNTER Kra 


\section{ADMISSIBLE MODULES AND A CHARACTERIZATION OF REDUCED LEFT ARTINIAN RINGS}

\section{GUENTER R. KRAUSE}

A unitary left $R$-module $M$ over a left noetherian ring $R$ with identity is called admissible if every prime ideal of $R$, which is the left annihilator of all nonzero submodules of a submodule $N$ of $M$, is also the left annihilator of all nonzero elements of $N$. The object of this paper is to study left noetherian rings $R$ whose category of unitary left $R$-modules consists of admissible modules. A ring $R$ in this class of rings is characterized by the fact that every injective left $R$ module is a direct sum of injective and indecomposable modules of the form $E_{R}(R / P)$, where $P$ denotes a prime ideal of $R$ and $E_{R}(R / P)$ the injective hull of the left $R$-module $R / P$.

Lesieur and Croisot have defined the heart $C(E)$ of an injective module $E$ to be the intersection of the kernels of all endomorphisms in the Jacobson radical of $\operatorname{Hom}_{R}(E, E)$, and the heart of any module $M$ to be the submodule $C(M)=M \cap C(E(M))$. Although the socle is always contained in the heart, the two submodules are not equal in general, a simple example being a torsion-free abelian group. This suggests the study of rings with the property that heart and socle coincide in every one of their modules. In $\S 3$ the discussion is restricted to left artinian rings, whose left modules are admissible, and it is shown that the class of these rings is the class of all reduced left artinian rings, a ring being reduced if it is a direct sum of division rings modulo its Jacobson radical. A ring $R$ in this class has the interesting property that for every prime ideal $P$ the heart of the left $R$-module $R / P$ is equal to its socle. But, although these modules form an injective decomposition basis for the category of all unitary left $R$-modules, socle and heart can be different for some objects in this category. In $\S 4$, however, it is shown that socle and heart coincide in every module over a reduced left artinian ring $R$ if and only if $R$ is a direct sum of finitely many local left artinian rings. This result admits two interesting corollaries:

1. A commutative noetherian ring with identity is artinian if and only if heart and socle are equal for every left $R$ module.

2. A left noetherian ring with identity whose modules are admissible and have coinciding heart and socle has the Artin-Rees property for left ideals.

1. Preliminaries. Throughout this paper each ring $R$ will be a (not necessarily commutative) ring with identity element 1 , and each module $M$ will be a unitary left $R$-module in the sense that $1 m=m$ 
for all $m \in M$. The category of all left $R$-modules is denoted by ${ }_{R} \mathscr{L}$, a module $M$ is called a cogenerator in ${ }_{R} \mathscr{C l}$ if every module of ${ }_{R} \mathscr{C l}$ is isomorphic to a submodule of a direct product of copies of $M$. If $M \in_{R} \mathscr{C l}$, then $E(M)$ or $E_{R}(M)$ denotes the injective hull of $M$. It is well-known (see [15]) that an injective module $E$ over a left noetherian ring $R$ is a direct sum of injective and (directly) indecomposable left $R$-modules. Furthermore, the direct summands appearing in such a decomposition are unique up to an automorphism of $E$. Thus, the set of all isomorphism classes of indecomposable injective submodules of the injective hull $E(M)$ of a module $M$ is an invariant of $M$, and we denote by $\pi(M)$ a set of representatives of these classes. A module $M$ is said to be homogeneous if $|\pi(M)|=1, E$-homogeneous if $\pi(M)=\{E\}$. A left ideal $L$ of $R$ is called homogeneous if the left $R$-module $R / L$ is homogeneous.

A submodule $N$ of $M$ is called large in $M$ (denoted by $N \cong \subseteq^{\prime} M$ ), and $M$ is said to be an essential extension of $N$ if $N \cap X \neq 0$ for every nonzero submodule $X$ of $M$. Dually, a submodule $N$ of $M$ is small in $M$ if $X+N \neq M$ for all proper submodules $X$ of $M . \quad M$ is uniform if every nonzero submodule is large in $M$, a left ideal $L$ is irreducible if the left $R$-module $R / L$ is uniform. $L$ is said to be completely irreducible if the intersection of all left ideals properly containing $L$ is different from $L$. A left ideal $L$ is an irredundant intersection of left ideals $L_{i}, i \in I$, if $L=\bigcap_{i \in I} L_{i}$ but $L \neq \bigcap_{i \neq j} L_{i}$ for every $j \in I$.

$X_{\ell}=X_{\ell}(R)=\{r \in R \mid r X=0\}$ denotes the left annihilator in $R$ of the subset $X$ of $M, Y_{r}(M)=\{m \in M \mid Y m=0\}$ denotes the right annihilator in $M$ of the subset $Y$ of $R$. A prime ideal $P$ is called associated with $M$, if $P=N_{\ell}^{\prime}$ for all nonzero submodules $N^{\prime}$ of some submodule $N$ of $M$, it is called strictly associated with $M$ if furthermore $P=n_{\ell}$ for all $0 \neq n \in N$. The set of all prime ideals associated with $M$ is called the characteristic of $M$ and denoted by ch $(M)$, the set of all prime ideals $P$ with $P \supseteqq M_{\ell}$ is denoted by $\operatorname{supp}(M)$. A prime ideal $P$ is said to be completely prime if the ring $R / P$ has no zero-divisors. An ideal $K$ is called corpoidal if $R / K$ is a division ring.

$J=J(R)=$ Jacobson radical of the ring $R$.

$N=N(R)=$ Baer lower nil radical of $R$.

$N^{*}=N^{*}(R)=$ generalized nil radical $=$ intersection of all completely prime ideals.

$T=T(R)=$ Thierrin radical $=$ intersection of all corpoidal ideals.

$\bigoplus_{i \in I} M_{i}=$ (discrete) direct sum of the modules $M_{i}, i \in I$.

$\Pi_{i \in I} M_{i}=$ direct product (complete direct sum) of the modules $M_{i}, i \in I$. 
$S(M)=$ socle of the module $M=$ sum of all minimal submodules of $M$.

$Z(M)=$ singular submodule of $M=\left\{m \in M \mid m_{\ell} \varrho^{\prime} R\right\}$.

$C(M)=$ heart of $M=M \cap \bigcap_{\beta \in J\left(\mathrm{Hom}_{R}(E(M), E(I))\right)} \operatorname{ker} \beta$.

$Z=$ ring of integers.

$\operatorname{dim}_{R} M=$ Goldie-dimension of the module $M$ (see [7] and [8]).

The tertiary radical ter $L$ of a left ideal $L$ is the set of all elements $r \in R$ such that for every $b \notin L$ there exists an element $a \in R b$, $a \notin L$ with $r R a \leqq L$. The radical $\operatorname{rad} L$ of $L$ is the intersection of all prime ideals containing $(R / L)_{\ell}(R)$, that is, $\operatorname{rad} L /(R / L)_{\ell}=$ $N\left(R /(R / L)_{\ell}\right) . \quad L$ is called tertiary if every element which annihilates a nonzero submodule of $R / L$ belongs to ter $L$, it is primary, if every such element belongs to $\operatorname{rad} L$. If $L$ is tertiary, then ter $L$ is a prime ideal (see [4]). Since $\operatorname{rad} L$ is always contained in ter $L$, primary left ideals are tertiary, the converse, however, is not true in general. Furthermore, in general $L$ is neither contained in $\operatorname{rad} L$ nor in ter $L$. It is well-known that all tertiary left ideals of a left noetherian ring $R$ are primary if and only if $R$ has the Artin-Rees-property for left ideals, that is, for every left ideal $L$, every two-sided ideal $I$, and every nonnegative integer $n$ there exists a nonnegative integer $m=$ $m(L, I, n)$ such that $I^{m} \cap L \subseteq I^{n} L$. Finally, we call a ring $R$ local if it has exactly one maximal left ideal, which is equivalent to the fact that $R / J$ is a division ring.

2. Admissible and strictly admissible modules. In [11] it was shown that submodules, injective hulls and direct sums of admissible modules over a left noetherian ring are admissible. In general, however, epimorphic images of admissible modules are not necessarily admissible. This follows from [11, Folgerung 2.8] and [12, Example 10.1].

Definition 2.1. A module $M$ is strictly admissible if $M$ and its homomorphic images are admissible.

An ascending series of submodules of the module $M$ is a set of submodules $\left\{N_{\alpha} \mid \alpha \in A\right\}$ with the following properties: (1) $N_{0}=0$ and $N_{\mathfrak{S}}=M$ for some $\subseteq \in A$. (2) $N_{\alpha} \subseteq N_{\alpha+1}$ for each $\alpha<\mathfrak{S}$. (3) $N_{\lambda}=$ $\mathrm{U}_{\alpha<\lambda} N_{\alpha}$ for limit ordinals $\lambda$.

Proposition 2.2. The following properties of the module $M$ over the left noetherian ring $R$ are equivalent:

(1) $M$ is strictly admissible.

(2) If $N$ is a homomorphic image of $M$, then there exists an ascending series $\left\{N_{\alpha} \mid \alpha \in A\right\}$ of submodules of $N$ and a set $\left\{P_{\alpha} \mid \alpha \in A\right\}$ 
of completely prime ideals of $R$ such that $N_{\alpha+1} / N_{\alpha} \cong R / P_{\alpha}$ for all $\alpha \in A$.

(3) Every nonzero homomorphic image of $M$ has a nonzero admissible submodule.

Proof. (1) $\rightarrow(2)$ : Let $N$ be a homomorphic image of $M$, and let $N_{0}=0$. If $\alpha>0$ is not a limit ordinal and $N_{\alpha-1} \neq N$, then $\operatorname{ch}\left(N / N_{\alpha-1}\right) \neq \varnothing$ by [11, Lemma 1.9]. Since $M$ is strictly admissible, there exists a completely prime ideal $P_{\alpha-1}$ of $R$ such that $P_{\alpha-1}=(R r \bar{x})_{\ell}=(r \bar{x})_{\ell}$ for an element $0 \neq \bar{x} \in N / N_{\alpha-1}$ and all $r \notin \bar{x}_{\ell}$ (see [11, Satz 2.6]). If $\bar{x}=$ $x+N_{\alpha-1}$, define $N_{\alpha}=N_{\alpha-1}+R x$, whence $N_{\alpha} / N_{\alpha-1} \simeq R \bar{x} \simeq R / \bar{x} \ell=$ $R / P_{\alpha-1}$. If $\lambda$ is a limit ordinal, we define $N_{\lambda}=\bigcup_{\alpha<\lambda} N_{\alpha}$. Then there exists an index $\subseteq$ such that $N_{\Im}=N_{\Im^{+1}}$, whence $\operatorname{ch}\left(N / N_{\Im}\right)=\varnothing$, and thus $N=N_{\subseteq}$ since $R$ is left noetherian.

$(2) \rightarrow(3):$ If $N$ is a nonzero homomorphic image of $N$ and $\left\{N_{\alpha} \mid \alpha \in A\right\}$ an ascending series of submodules of $N$ of the type described in (2), let $N_{\alpha_{0}}$ denote the first nonzero element of this series. It is clear that $\alpha_{0}$ is not a limit ordinal, and hence $N_{\alpha_{0}-1}=0$. Thus $N_{\alpha_{0}}=$ $N_{\alpha_{0}} / N_{\alpha_{0}-1} \simeq R / P_{\alpha_{0}-1}$, and since $P_{\alpha_{0}-1}$ is completely prime, $N_{\alpha_{0}}$ is admissible.

$(3) \rightarrow(1)$ : Let $N$ be a nonzero homomorphic image of $M$, and let $K$ be a maximal nonzero admissible submodule of $N$. The existence of $K$ is guaranteed by (3) and Zorn's lemma. If $K \neq N$, then there exists a nonzero admissible submodule $L / K$ of $N / K$. If $K \cong \subseteq^{\prime} L$, then $E(K)=E(L)$, so $L$ is admissible by [11, Satz 2.6], a contradiction to the maximality of $K$. If $H \subseteq L$ with $H \cap K=0$, then $H \simeq(K \oplus H) /$ $K \subseteq L / K$, so $K \oplus H$ is admissible by [11, Lemma 2.5], which also contradicts the maximality of $K$. Hence $K=N$, and $N$ is admissible.

REMARK 2.3. If $M$ has an ascending series of the type described in 2.2, then $\operatorname{ch}(M) \subseteq\left\{P_{\alpha} \mid \alpha \in A\right\} \subseteq \operatorname{supp}(M)$. The first inclusion follows from the fact that for every $P \in \operatorname{ch}(M)$ there exists a smallest index $\alpha$ such that $P \in \operatorname{ch}\left(N_{\alpha}\right)$. Since $\alpha$ is not a limit ordinal, $P \in \operatorname{ch}\left(N_{\alpha} / N_{\alpha-1}\right) \cup \operatorname{ch}\left(N_{\alpha-1}\right)$, whence $P \in \operatorname{ch}\left(N_{\alpha} / N_{\alpha-1}\right)=\left\{P_{\alpha-1}\right\} . \quad$ The second inclusion follows from $\left(N_{\alpha+1} / N_{\alpha}\right)_{\ell} \supseteq M_{\ell}$ for all $\alpha \in A$.

In general, however, $\operatorname{ch}(M) \neq\left\{P_{\alpha} \mid \alpha \in A\right\} \neq \operatorname{supp}(M)$. If, for example $p_{i}$ denotes the $i$-th prime number and $\left(p_{i}\right)$ the ideal of $Z$ generated by $p_{i}$, then $M=\bigoplus Z /\left(p_{i}\right)$ is a strictly admissible $Z$-module with ascending series $N_{k}=\bigoplus_{i=1}^{k} Z /\left(p_{i}\right)$ and $N_{\omega}=\bigcup_{k=1}^{\infty} N_{k}=M$. Since $M_{\ell}=0,\left\{\left(p_{i}\right) ; i=1,2, \cdots\right\} \neq \operatorname{supp}(M)$. On the other hand, the left $Z$-module $Z$ has the ascending series $0 \subset 2 Z \subset Z$ with factors $Z /(2)$ and $Z /(0)$, whereas $\operatorname{ch}(Z)=\{(0)\}$. In general, however, the following is true: 
Proposition 2.4. The following properties of the module $M$ over the left noetherian ring $R$ are equivalent:

(a) $M$ is strictly admissible.

(b) If $\left\{N_{\alpha} \mid \alpha \in A\right\}$ is an ascending series of submodules of $M$ and $\left\{P_{\alpha} \mid \alpha \in A\right\}$ a set of prime ideals of $R$ such that $N_{\alpha+1} / N_{\alpha} \simeq R / P_{\alpha}$ for every $\alpha \in A$, then $\left\{P_{\alpha} \mid \alpha \in A\right\}=$ $\operatorname{ch}(M)$.

(2) $\{$ (a) $M$ is admissible.

(b) $\pi(M / N) \cap \pi(M) \neq \varnothing$ for all submodules $N$ of $M$.

(3) $\{$ (a) $M$ is admissible.

(b) $\pi(M / N) \subseteq \pi(M)$ for all submodules $N$ of $M$.

(a) $M$ is strictly admissible.

(b) $\operatorname{ch}(M / N) \subseteq \operatorname{ch}(M)$ for all submodules $N$ of $M$.

Proof. The equivalence of (3) and (4) is an immediate consequence of [11, Folgerung 2.8].

$(1) \rightarrow(2)$ : Since a submodule $N$ of a strictly admissible module $M$ is strictly admissible, there exists an ascending series of submodules $\left\{N_{\alpha} \mid \alpha \in A\right\}$ of $N$ and a set $\left\{Q_{\alpha} \mid \alpha \in A\right\}$ of prime ideals such that $N_{\alpha+1} / N_{\alpha} \simeq R / Q_{\alpha}$ for all $\alpha \in A$. Similarly, there exists an ascending series $\left\{K_{\beta} / N \mid \beta \in B\right\}$ of submodules of $M / N$ and a set $\left\{P_{\beta} \mid \beta \in B\right\}$ of prime ideals such that $K_{\beta+1} / K_{\beta} \simeq R / P_{\beta}$ for all $\beta \in B$. Thus, the series $0=$ $N_{0} \subset N_{1} \subset \cdots \subset N_{\alpha} \subset N_{\alpha+1} \subset \cdots \subset N=K_{0} \subset K_{1} \subset \cdots \subset K_{\beta} \subset K_{\beta+1} \subset \cdots \subset M$ is an ascending series of submodules of $M$ whose factors are isomorphic to $R / Q_{\alpha}$ and $R / P_{\beta}$, respectively. Since $\left\{Q_{\alpha} \mid \alpha \in A\right\} \cup\left\{P_{\beta} \mid \beta \in B\right\}=\operatorname{ch}(M)$ by assumption, it follows in particular that $P_{0}=\left(K_{1} / N\right)_{\ell} \in \operatorname{ch}(M)$. Thus $M$ and $M / N$ both contain a submodule isomorphic to $R / P_{0}$, whence $\pi(M / N) \cap \pi(M) \neq \varnothing$.

(2) $\rightarrow(4)$ : Let $N \subseteq M$ and define $N_{0}=N$. If $\alpha>0$ is not a limit ordinal, let $X \in \pi\left(M / N_{\alpha-1}\right) \cap \pi(M)$, and let $E$ be a submodule of $E\left(M / N_{\alpha-1}\right)$ isomorphic to $X$. Since $M$ is admissible, it follows from [11, Satz 2.6] that $E \simeq E_{R}\left(R / P_{\alpha-1}\right)$ for some $P_{\alpha-1} \in \operatorname{ch}(M) \cap \operatorname{ch}\left(M / N_{\alpha-1}\right)$. Since $E$ is admissible, and since $\operatorname{ch}\left(M / N_{\alpha-1} \cap E\right)=\left\{P_{\alpha-1}\right\}, M / N_{\alpha-1} \cap E$ possesses a submodule $N_{\alpha} / N_{\alpha-1}$ which is isomorphic to $R / P_{\alpha-1}$. If $N_{\lambda}=\mathrm{U}_{\alpha<\lambda} N_{\alpha}$ for limit ordinals $\lambda$, it follows that the factors of the ascending series

$$
0=N_{0} / N \subset N_{1} / N \subset \cdots \subset N_{\alpha} / N \subset N_{\alpha+1} / N \subset \cdots \subset M / N
$$

are isomorphic to modules $R / P_{\alpha}$, where every $P_{\alpha}$ is associated with $M$ and hence completely prime. Thus, $M$ is strictly admissible and it follows from the construction that $\operatorname{ch}(M / N) \leqq\left\{P_{\alpha} \mid \alpha \in A\right\} \subseteq \operatorname{ch}(M)$.

$(4) \rightarrow(1)$ : If $\left\{N_{\alpha} \mid \alpha \in A\right\}$ is an ascending series of submodules of $M$ with factors $R / P_{\alpha}$, it follows that $P_{\alpha} \in \operatorname{ch}\left(M / N_{\alpha}\right) \subseteq \operatorname{ch}(M)$ for every $\alpha \in A$. Together with 2.3 this implies (1b). 
CoROLlaRY 2.5. If $M$ is a module with the properties (1) to (4) in 2.4, then $S(M)=C(M)=\oplus P_{r}(M)$, where $P$ runs through the set of maximal elements of $\operatorname{ch}(M)$.

Proof. Since $S(M)$ is the intersection of all large submodules of $M$ (see [17, p. 23]), $S(M) \subseteq C(M)$. Since $\pi(M / N) \subseteq \pi(M)$ for all submodules $N$ of $M$, it follows from [11, Satz 3.1] that $C(M)=S(M)$. By [11, Lemma 3.5], $P_{r}(M) \cong C(M)$ for every maximal element $P$ of ch $(M)$. Conversely, if $R x$ is a minimal submodule of $M$, then $P=$ $(R x)_{\ell} \in \operatorname{ch}(M)$, whence $P=x_{\ell}$ by the admissibility of $M$. Thus, $P$ is a maximal element of $\operatorname{ch}(M)$. Since $R x \leqq(R x)_{\ell_{r}}(M)=P_{r}(M)$, and since $S(M)$ is the sum of all minimal submodules of $M$, the second equation thus follows from the first.

REMARK 2.6. The author does not know whether a strictly admissible module $M$ with $S(M)=C(M)$ also has property $2.4(1 \mathrm{~b})$. If, however, all left $R$-modules $M$ over the left noetherian ring $R$ are admissible and satisfy $S(M)=C(M)$, then $\operatorname{ch}(M)=\operatorname{supp}(M)$ for all $M$, which implies $2.4(1 \mathrm{~b})$. This is shown in $\S 4$.

THEOREM 2.7. The following properties of the left noetherian ring $R$ are equivalent:

(1) Every left $R$-module is admissible.

(2) $R / N$ is a strictly admissible left $R$-module.

(3) Every injective indecomposable left $R$-module $E$ is isomorphic to $E_{R}(R / P)$ for some prime ideal $P$.

(4) $R /$ ter $L$ is $R$-isomorphic to a submodule of $R / L$ for every irreducible left ideal $L$.

(5) Every tertiary left ideal $L$ is $E_{R}(R /$ ter $L)$-homogeneous.

Proof. Trivially, (2) follows from (1). Assume (2), and let $E$ be an injective indecomposable left $R$-module with $\operatorname{ch}(E)=\{P\}$. Then $P=(R r x)_{\iota}$ for some element $0 \neq x \in E$ and every $r \notin x_{\iota}$. Since $R x \simeq$ $R / x_{\ell} \simeq(R / N) /\left(x_{\ell} / N\right), R x$ is admissible. Thus, $E$ is admissible by [11, Hilfssatz 2.4], whence $P=x_{\ell}$ which implies $E=E(R x) \simeq E_{R}\left(R / x_{\ell}\right)=$ $E_{R}(R / P)$.

$(3) \rightarrow(4)$ : Since $E_{R}(R / L)$ is injective and indecomposable for every irreducible left ideal $L$, it follows from (3) that $E_{R}(R / L) \simeq E_{R}(R / P)$ for some prime ideal $P$. Since $R / P$ is a left noetherian left uniform prime ring, $P$ is completely prime by [7, Lemma 3.3 and Lemma 3.8]. This implies the admissibility of $R / L$ by [11, Hilfssatz 2.4]. Since $L$ is irreducible, $L$ is tertiary, and since $\operatorname{ch}(R / L)=\{P\}$, it follows that ter $L=P$. Since $P=x_{\ell}$ for some element $0 \neq x \in R / L$ by the admissibility of $R / L$, it follows that $R /$ ter $L=R / P=R / x_{\ell} \simeq R x \leqq R / L$. 
$(4) \rightarrow(5)$ : Let $L$ be tertiary, $L=\bigcap_{i=1}^{n} L_{i}$ with irreducible left ideals $L_{i}$, none of which is superfluous. It follows from [15, Th. 2.3] that $E(R / L) \simeq \bigoplus_{i=1}^{n} E\left(R / L_{i}\right)$, and since each $L_{i}$ is ter $L$-tertiary by [12, Th. 8.3], it follows that $E\left(R / L_{i}\right) \simeq E(R /$ ter $L)$ for each $i$.

$(5) \rightarrow(1)$ : Let $E$ be injective and indecomposable with $\operatorname{ch}(E)=\{P\}$, and let $x \neq 0$ be an element of $E$. Since $x_{\ell}$ is a tertiary left ideal with ter $x_{\ell}=P$, it follows from (5) that $E=E(R x) \simeq E\left(R x_{\ell}\right) \simeq$ $E_{R}(R / P)$. Since $R / P$ has no zero-divisors by [7, Lemma 3.3 and 3.8], $E$ is admissible. Since $R$ is left noetherian, every injective left $R$ module is a direct sum of indecomposable injective modules (see [15, Th. 2.5]), and since direct sums of admissible modules are admissible (see [11, Lemma 2.5]), it follows that every left $R$-module is admissible.

REMARK 2.8. In 2.7 (5) one cannot simply demand the homogeneity of all tertiary left ideals. By [12, Propriété 10.40] every left artinian ring has this property, but not every module over a left Artinian ring is admissible (see [11, Hilfssatz 4.1]).

3. A characterization of reduced left artinian rings. Following [2], we call a ring $R$ reduced if $R / J$ is a direct sum of division rings. These rings deserve some interest, since the problem of constructing all rings with minimum condition on left ideals can be reduced completely to that of constructing all reduced rings with minimum condition on left ideals (see [2] and [9]). The purpose of this section is to give a characterization of reduced left artinian rings by certain properties of the category of their unitary left modules.

Proposition 3.1. The following properties of a prime ideal $P$ of the left noetherian ring $R$ are equivalent:

(1) $P$ is a completely irreducible left ideal.

(2) Every left $R$-module $M$ with $\operatorname{ch}(M)=\{P\}$ is admissible and has nonzero socle.

(3) $P$ is a maximal left ideal.

(4) $\{$ (a) $P$ is completely prime.

(b) $S_{R}(R / P) \neq 0$.

Proof. $(1) \rightarrow(2)$ : Since $R / P$ is uniform, $S_{R}(R / P)$ is the intersection of all nonzero submodules of $R / P$ and hence nonzero, since $P$ is completely irreducible. If $M$ is a left $R$-module with $\operatorname{ch}(M)=\{P\}$, then $E(M)=\bigoplus_{i \in I} E_{i}$ with injective indecomposable left $R$-modules $E_{i}$ (see [15, Th. 2.5]). By [11, Folgerung 1.11 and 1.13], $\operatorname{ch}\left(E_{i}\right)=\{P\}$ for all $i \in I$. Since $R$ is left noetherian, $C\left(E_{i}\right) \neq 0$ by [13, Propriété 3.3], whence $E_{i} \simeq E\left(C\left(E_{i}\right)\right.$ ) for every $i$. Hence, there exists a nonzero 
element $x \in C\left(E_{i}\right)$ such that $P=(R r x)_{\ell}$ for all $r \notin x /$. Since $P=$ $\bigcap_{r \in R}(r x)_{\ell}$, and since $P$ is completely irreducible, $P=\left(r_{0} x\right)_{\ell}$ for some $r_{0} \in R$. By [13, Propriété 3.2], $\left(r_{0} x\right)_{\ell}$ is maximal in the set of all left annihilators of nonzero elements in $E_{i}$, so that $P=\left(r_{0} x\right)_{\ell}=$ $(R x)_{\ell} \subseteq(r x)_{\ell}$ implies $P=(r x)_{\ell}$ for all $r \notin x_{\ell}$. Thus, $C\left(E_{i}\right)$ is admissible, and so is $E_{i}$ by [11, Hilfssatz 2.4]. Therefore $M$ is admissible by [11, Lemma 2.5]. Furthermore, $S(M) \neq 0$ since $S_{R}(R / P) \neq 0$ and $E_{i}=E\left(R r_{0} x\right) \simeq E\left(R /\left(r_{0} x\right)_{\ell}\right)=E(R / P)$ for all $i \in I$.

$(2) \rightarrow(3)$ : Since $\operatorname{ch}(R / P)=\{P\}$, the left $R$-module $R / P$ is admissible and has nonzero socle. Hence there exists a nonzero element $x \in S(R / P)$ such that $P=x_{\ell}$. Since $P$ is completely prime by [11, Satz 2.6], the ring $R / P$ is a left noetherian ring without zero-divisors and therefore left uniform by [8, Proposition 4.2]. Thus, $S(R / P)=$ $R x \simeq R / x_{\ell}=R / P$, and since $S(R / P)$ is a simple left $R$-module, $P$ is a maximal left ideal.

It is obvious that (4) follows from (3).

$(4) \rightarrow(1)$ : Since $P$ is completely prime, $P$ is an irreducible left ideal by [8, Proposition 4.2], and hence $S(R / P)$ is the intersection of all nonzero submodules of $R / P$. Since $S(R / P) \neq 0$ by assumption, it follows that $P$ is completely irreducible.

Proposition 3.2. If $I$ is a semi-prime ideal of the ring $R$ with maximum condition on left ideals, then:

(a) $R / I$ is an irredundant (see [14]) subdirect sum of finitely many prime rings $\bar{R}_{i}=R / P_{i}, i=1, \cdots, n$.

(b) $\operatorname{ch}_{R}(R / I)=\left\{P_{1}, \cdots, P_{n}\right\}$ and $P_{i} \not P_{j}$, if $i \neq j$.

(c) $I=\bigcap_{i=1}^{n} P_{i}$.

Proof. By [14, Th. 3.2], $\bar{R}=R / I$ is the irredundant subdirect sum of prime rings $\bar{R}_{\alpha}, \alpha \in A$, and the kernels of the projections $\bar{R} \rightarrow \bar{R}_{\alpha}$ are the maximal annihilator ideals $\bar{P}_{\alpha}$ of $\bar{R}$. The proof of Theorem 3.2 in [14] shows also that $\bigcap_{\alpha \in A} \bar{P}_{\alpha}=0$. If $\bar{P}_{\alpha}=P_{\alpha} / I$ for an ideal $P_{\alpha}$ of $R$, then every $P_{\alpha}$ is a prime ideal of $R$. Let $\bar{P}_{\alpha}=$ $(L / I)_{\ell}(\bar{R})$ for some left ideal $L$ of $R$, and let $I \subset L_{1} \subseteq L$. If $\varphi$ denotes the canonical epimorphism from $R$ onto $\bar{R}, 0=\left(P_{\alpha} / I\right) \cdot\left(L_{1} / I\right)=P_{\alpha} \varphi \cdot L_{1} \varphi=$ $\left(P_{\alpha} L_{1}\right) \varphi$, whence $P_{\alpha} \subseteq\left(L_{1} / I\right)_{\ell}(R)$. If $a L_{1} \subseteq I$, then $(a \varphi)\left(L_{1} \varphi\right)=0$, and hence $a \varphi \in\left(L_{1} / I\right)_{\ell}(\bar{R})=P_{\alpha} / I$ which implies $a \in P_{\alpha}$, since $\operatorname{ker} \varphi=I$. Thus, $P_{\alpha}=\left(L_{1} / I\right)_{\ell}(R)$, whence $P_{\alpha} \in \operatorname{ch}_{R}(R / I)$. Since $\bar{P}_{\alpha}$ is a maximal annihilator ideal in $\bar{R}, P_{\alpha}$ is maximal in $\operatorname{ch}_{R}(R / I)$. Since $\operatorname{ch}_{R}(R / I)$ is finite by the maximum condition on left ideals in $R, A$ is finite. Since $I=\bigcap_{\alpha \in A} P_{\alpha}$, and since every ideal associated with the left $R$-module $R / I$ contains $I, \operatorname{ch}_{R}(R / I)=\left\{P_{\alpha} \mid \alpha \in A\right\}$.

Definition 3.3. (See $[10$, p. 216].) A set $\phi$ of prime ideals $P$ 
of a semi-prime ring $R$ is a minimal decomposition set for $R$ if $\bigcap_{P \in \phi} P=0$ but $\bigcap_{P \in \phi-X} P \neq 0$ for all $X \in \dot{\phi}$.

If $\phi$ denotes the set of all prime ideals of $R$, then 3.2 yields the following result:

COROLLARY 3.4. The following properties of the left noetherian ring $R$ are equivalent:

(1) Prime ideals of $R$ are maximal ideals.

(2) The prime ideals of $R / N$ form a minimal decomposition set for $R / N$.

(3) $|\dot{\phi}| \leqq|\pi(R / N)|$.

Proof. (1) $\rightarrow(2)$ : Since every left ideal of a left noetherian ring $R$ contains a product of finitely many prime ideals (see [12, Th. 3.1]), $R$ has only finitely many prime ideals $P_{1}, \cdots, P_{n}$. Since they are maximal, and since their intersection is $N$, the prime ideals of $R / N$ form a minimal decomposition set for $R / N$.

$(2) \rightarrow(3)$ : Since $N$ is the intersection of all prime ideals associated with the left $R$-module $R / N$ (see 3.2), and since the prime ideals of $R / N$ form a minimal decomposition set for $R / N$, it follows that $\phi=$ $\operatorname{ch}_{R}(R / N)$, whence $|\dot{\phi}|=\left|\operatorname{ch}_{R}(R / N)\right| \leqq|\pi(R / N)|$ by [11, Hilfssatz 1.15].

$(3) \rightarrow(1):$ By 3.2, $N=\bigcap_{i=1}^{n} P_{i}$, where $\operatorname{ch}_{R}(R / N)=\left\{P_{1}, \cdots, P_{n}\right\}$. Thus, $R / N$ can be imbedded in the module $\bigoplus_{i=1}^{n} R / P_{i}$. Since $\pi\left(R / P_{i}\right) \neq$ $\pi\left(R / P_{j}\right)$ for $i \neq j$, and since for every prime ideal $P$ the left $R$-module $R / P$ is homogeneous, it follows that

$$
|\phi| \leqq|\pi(R / N)| \leqq\left|\pi\left(\bigoplus_{i=1}^{n} R / P_{i}\right)\right|=n=|\operatorname{ch}(R / N)| \leqq|\phi| .
$$

Together with $\phi \supseteqq \operatorname{ch}_{R}(R / N)$ and the finiteness of $\operatorname{ch}_{R}(R / N)$ this implies $\operatorname{ch}_{R}(R / N)=\phi$, and therefore prime ideals are maximal ideals by 3.2 .

COROLLARY 3.5. The following properties of the left noetherian ring $R$ are equivalent:

(1) Every prime ideal of $R$ is corpoidal.

(2) $|\phi| \leqq|\pi(R / T)|$.

Proof. (1) implies $T=N$, and thus $|\phi| \leqq|\pi(R / N)|=|\pi(R / T)|$ by 3.4. Conversely, $|\phi| \leqq|\pi(R / T)|$ implies the finiteness of $\phi$, since $\operatorname{dim}_{R}(R / T)<\infty$. Thus, $T$ is the intersection of a finite number of corpoidal ideals $K_{1}, \cdots, K_{n}$ and none of these is superfluous. Thus, by [15, Th. 2.3], $E(R / T) \simeq \bigoplus_{i=1}^{n} E\left(R / K_{i}\right)$, whence 


$$
\begin{aligned}
|\phi| & \leqq\left|\pi\left(E_{R}(R / T)\right)\right| \\
& =\left|\left\{E_{R}\left(R / K_{1}\right), \cdots, E_{R}\left(R / K_{n}\right)\right\}\right|=n=|\operatorname{ch}(R / T)| \leqq|\phi| .
\end{aligned}
$$

Hence $\phi=\operatorname{ch}_{R}(R / T)$, and therefore all prime ideals of $R$ are corpoidal.

THEOREM 3.6. The following properties of the ring $R$ are equivalent:

(1) Every left $R$-module can be imbedded in a direct sum of copies of the left $R$-module $E_{R}\left(R / N^{*}\right)$.

(2) $\{$ (a) $R$ is left noetherian.

(c) $S(R / P)=C(R / P)$ for every prime ideal $P$ of $R$.

(3) $\left\{\begin{array}{l}\text { (a) } R \text { is left artinian. } \\ \text { (b) Every left ideal containing } J \text { is two-sided. }\end{array}\right.$

(4) $\{$ (b) $E$ and $\oplus$ commute.

(c) $E_{R}\left(R / N^{*}\right)$ is a cogenerator in the category ${ }_{R} \mathscr{l l}$.

(5) $\left\{\begin{array}{l}\text { (a) } R \text { is left noetherian. } \\ \text { (b) Prime ideals of } R \text { are maximal left ideals. }\end{array}\right.$

(a) $T=N$.

(6) $\{$ (b) The number of prime ideals of $R$ is finite.

(c) $R$ has the maximum condition on nil left ideals.

(a) $R$ has the minimum condition on principal left ideals.

(7) $\{$ (b) A left ideal is small if and only if it is generated by finitely many nilpotent elements.

(8) $R$ is a reduced left artinian ring.

Proof. (1) $\rightarrow(2)$ : If $d$ denotes the cardinality of $E_{R}\left(R / N^{*}\right)$, then every module is contained in a direct sum of modules generated by $d$ elements. By [5, Th. 3.3], this implies the maximum condition on left ideals. Since $R / N^{*}$ is semi-prime, $N^{*}$ is the intersection of the prime ideals $P_{1}, \cdots, P_{n}$ associated with the left $R$-module $R / N^{*}$ (see Proposition 3.2). If $P$ is any prime ideal, then $P \in \operatorname{ch}(R / P) \subseteq$ $\operatorname{ch}\left(\oplus E\left(R / N^{*}\right)\right)=\operatorname{ch}\left(R / N^{*}\right)$, and so $\operatorname{ch}\left(R / N^{*}\right)$ is the set of all prime ideals of $R$. Therefore, every prime ideal is maximal by Proposition 3.2. Since $N^{*}$ is the intersection of all completely prime ideals, all prime ideals of $R$ are completely prime. Since completely prime ideals of a left noetherian ring are irreducible left ideals by [8, Proposition 4.2], it follows from [15, Th. 2.3] that $E\left(R / N^{*}\right) \simeq \bigoplus_{i=1}^{n} E\left(R / P_{i}\right)$. Since by [11, Hilfssatz 2.4 and Lemma 2.5] admissibility is inherited by injective hulls and direct sums, it follows that $E\left(R / N^{*}\right)$ is admissible, so all left $R$-modules are admissible by (1). Since prime ideals are maximal, it follows from [11, Hilfssatz 4.1] that prime ideals 
are maximal left ideals. Thus $S(R / P)=R / P=C(R / P)$ for all prime ideals $P$, since the heart of a nonzero module over a left noetherian ring is nonzero by [13, Propriété 3.3].

$(2) \rightarrow(3)$ : If $P$ is a prime ideal, it follows from (2) and [11, Lemma 3.5] that $R / P \supseteqq S(R / P)=C(R / P) \supseteqq P_{r}(R / P)=R / P$. By Proposition 2.2, there exists a sequence $0=L_{0} \subset L_{1} \subset \cdots \subset L_{n}=R$ of left ideals and a set of prime ideals $P_{0}, P_{1}, \cdots, P_{n-1}$ such that $L_{i} / L_{i-1} \simeq$ $R / P_{i-1}$ for all $i=1, \cdots, n$. Since $S\left(R / P_{i}\right)=R / P_{i}$, and since $R / P_{i}$ is left noetherian, each of the modules $R / P_{i}$ possesses a finite composition series, so $R$ is left artinian. It follows from Proposition 3.1 that every prime ideal is a maximal left ideal. Assume, not every left ideal containing $J$ is two-sided. Let $L$ be minimal in the set of all left ideals containing $J$ which are not the intersection of prime ideals. Since $R$ is left artinian, $J=N=\bigcap_{i=1}^{n} P_{i}$, where the $P_{i}$ denote the prime ideals of $R$. Let $M$ be maximal in the set of all left ideals containing $J$ and being properly contained in $L$. Then $M=P_{1} \cap \cdots \cap P_{m}$ with $m \leqq n$, and it may be assumed that $L \nsubseteq P_{1}$. Since $P_{1} \cap L=M$ and $P_{1}$ is a maximal left ideal, it follows that

$$
\begin{aligned}
R / L & =\left(P_{1}+L\right) / L \simeq P_{1} / P_{1} \cap L=P_{1} / M=P_{1} / P_{1} \cap \cdots \cap P_{m} \\
& \simeq\left[P_{1}+\left(P_{2} \cap \cdots \cap P_{m}\right)\right] / P_{2} \cap \cdots \cap P_{m}=R / P_{2} \cap \cdots \cap P_{m},
\end{aligned}
$$

whence $P_{2} \cap \cdots \cap P_{m}=(R / L)_{\ell} \subseteq L$. Since $P_{2} \cap \cdots \cap P_{m} \neq L$ by the choice of $L, P_{2} \cap \cdots \cap P_{m}=M \cong P_{1}$, contradicting the maximality of the prime ideals in $R$, and proving that every left ideal containing $J$ must be two-sided.

$(3) \rightarrow(4)$ : Since a left artinian ring with identity is left noetherian, direct sums of injective modules are injective, proving $(4 b)$. Since prime ideals of $R$ are maximal ideals, it follows from (3b) that they are maximal left ideals, so in particular completely prime. By 3.4, $R$ has only finitely many prime ideals $P_{1}, \cdots, P_{n}$, and therefore $N^{*}$ is their irredundant intersection. Thus, $E\left(R / N^{*}\right) \simeq \bigoplus_{i=1}^{n} E\left(R / P_{i}\right)$ by [8, Proposition 4.2] and [15, Th. 2.3]. If $M=R x \neq 0$ is a simple left $R$-module, then $x_{\ell}$ is a maximal left ideal and hence a prime ideal by (3b). Thus, $x_{\ell}=P_{i}$ for some $i$, whence $E(M) \simeq E\left(R / x_{\ell}\right) \subseteq E\left(R / N^{*}\right)$, which implies (4c) by [18, Lemma 1].

Let $\left\{M_{i} \mid i \in I\right\}$ be a family of left $R$-modules and let $I$ be wellordered. For $x=\prod_{i \in I} x_{i} \in \prod_{i \in I} E\left(M_{i}\right), x \notin \prod_{i \in I} M_{i}$, let $x_{i_{1}}$ be the first among the components $x_{i}$ of $x$ for which $x_{i} \notin M_{i}$, and let $L_{1}=$ $\left\{r \in R \mid r x_{i_{1}} \in M_{i_{1}}\right\}$. From $M_{i_{1}} \subseteq \subseteq^{\prime} E\left(M_{i_{1}}\right)$ it follows that there exists an element $r_{1} \in L_{1}$ such that $0 \neq r_{1} x_{i_{1}} \in M_{i_{1}}$. If, for $i>i_{1}$, there exists a component $x_{i}$ of $x$ such that $r_{1} x_{i} \notin M_{i}$ and $L_{1} \cap\left(x_{i}\right) / \subset\left\{r \in L_{1} \mid r x_{i} \in M_{i}\right\}$, let $x_{i_{2}}$ be the first such component, and let $L_{2}=\left\{r \in L_{1} \mid r x_{i_{2}} \in M_{i_{2}}\right\}$. Since $L_{2} x_{i_{2}} \neq 0$ and $M_{i_{2}} \cong E\left(M_{i_{2}}\right)$, there exists an element $r_{2} \in L_{2}$ such 
that $0 \neq r_{2} x_{i} \in M_{i_{2}}$. Continuing in this way, we obtain a strictly descending chain of left ideals $L_{n}, n=1,2, \cdots$. Since $R$ is left artinian, there exists a natural number $n>0$ such that for $i>i_{n}$ either $r_{n} x_{i} \in M_{i}$ or $L_{n} \cap\left(x_{i}\right)_{\ell}=\left\{r \in L_{n} \mid r x_{i} \in M_{i}\right\}$. Since $M_{i} \subseteq E\left(M_{i}\right)$, the second case implies $L_{n} x_{i}=0$, whence $r_{n} x_{i}=0$. By construction, $r_{n} x_{i} \in M_{i}$ for all $i \leqq i_{n}$ and $r_{n} x_{i_{n}} \neq 0$, so $0 \neq r_{n} x \in \prod_{i \in I} M_{i}$. Thus, $\prod_{i \in I} M_{i}$ is a large submodule of $\prod_{i \in I} E\left(M_{i}\right)$, which implies (4a).

$(4) \rightarrow(5)$ : It is well-known (see [3, Proposition 4.1]) that (5a) follows from (4b). By $3.2, N^{*}$ is the intersection of the prime ideals $P_{k}(k=1, \cdots, n)$, associated with the left $R$-module $R / N^{*}$, and this intersection is irredundant. If $P$ is any prime ideal, then $R / P \subseteq$ $\Pi E\left(R / N^{*}\right)=E\left(\Pi R / N^{*}\right)$ by $(4 \mathrm{~b})$ and $(4 \mathrm{c})$, whence $P \in \operatorname{ch}\left(\Pi R / N^{*}\right)$, and thus $P \supseteqq P_{k}$ for some $k$. Furthermore, there exists an element $x=\prod x_{i} \in \Pi R / N^{*}$ with $P=(R x)_{\ell}$, whence $P=\cap\left(R x_{i}\right)_{\ell} \leqq\left(R x_{i}\right)_{\ell}$ for all $i$, so $P$ is contained in one of the prime ideals associated with $R / N^{*}$. Thus, $P=P_{k}$, so $\operatorname{ch}_{R}\left(R / N^{*}\right)$ is the set of all prime ideals of $R$. Consequently, all prime ideals are maximal, and it follows from the definition of $N^{*}$ that they are also completely prime, so $E_{R}\left(R / N^{*}\right)$ is admissible by [15, Th. 2.3] and [11, Satz 2.6]. By assumption any module $M$ is isomorphic to a submodule of a direct product of copies of $E\left(R / N^{*}\right)$. If $P \in \operatorname{ch}(M), P=(R x)_{\ell}$ for some nonzero element $x=$ $\prod x_{i} \in M \sqsubseteq \Pi E\left(R / N^{*}\right)$. If $r \notin x_{\ell}$, then $r \notin\left(x_{i_{0}}\right) /$ for at least one component $x_{i_{0}}$ of $x$. Since $P$ is a maximal ideal, $P=\left(R s x_{i_{0}}\right) /$ for all $s \notin\left(x_{i_{0}}\right) /$, whence $P=\left(s x_{i_{0}}\right)$, since $E\left(R / N^{*}\right)$ is admissible. Thus, in particular $P=\left(r x_{i_{0}}\right)_{\ell}$, whence $P=(r x)_{\ell}$, since $\left(r x_{i_{0}}\right)_{\ell}=P \subseteq(r x)_{\ell}=$ $\cap\left(r x_{i}\right)_{\ell} \subseteq\left(r x_{i_{0}}\right)_{\ell}$. This implies the admissibility of $M$ and therefore (5b) by [11, Hilfssatz 4.1].

$(5) \rightarrow(6)$ : Since all prime ideals are corpoidal, $T=N$ follows from (5b). (6b) follows from (5b) by means of Corollary 3.4, and (6c) is weaker than (5a).

$(6) \rightarrow(7)$ : It is clear that $N=J=T$, and since $R$ has only finitely many prime ideals, all prime ideals are corpoidal. If the prime ideals of $R$ are denoted by $P_{1}, \cdots, P_{n}$, then the factors of the sequence

$$
J=P_{1} \cap \cdots \cap P_{n} \subset P_{1} \cap \cdots \cap P_{n-1} \subset \cdots \subset P_{1} \cap P_{2} \subset P_{1} \subset R
$$

are simple left $R$-modules, so $R / J$ is a semi-simple ring with minimum condition. Since every left ideal contained in $J$ is nil, it follows from (6c) that $J$ is a noetherian left $R$-module, so $R$ is left noetherian. Thus, $J$ is nilpotent, and therefore in particular $T$-nilpotent in the sense of Bass (see [1]). This implies (7a) by [1, Th. P]. By [16, Hilfssatz 3.5], every small left ideal is contained in $J$ and hence nil. Thus, a small left ideal is generated by finitely many nilpotent elements because of (6c). Conversely, it follows from $N=T$ that every 
nilpotent element is contained in $N$, and therefore it generates a nil left ideal. Thus, every left ideal generated by a finite number of nilpotent elements is small by [16, Satz 3.7].

$(7) \rightarrow(8)$ : By [20, Satz 1], $J$ is nil. Thus, if $n$ is nilpotent modulo $J, n$ is nilpotent, whence $R n$ is small and thus contained in $J$ by [16, Hilfssatz 3.5]. Thus, $R / J$ has no nonzero nilpotent elements, and since $R / J$ has minimum condition on principal left ideals, it follows from [6] that $R / J$ is a direct sum of division rings. By [16, Satz 3.7], every left ideal contained in $J$ is small and therefore finitely generated. Thus, $J$ is a noetherian left $R$-module, which implies the maximum condition on left ideals in $R$, since $R / J$ is semi-simple with minimum condition by [1, Th. P]. Therefore, $J$ is nilpotent, and since $J^{i} / J^{i+1}$ is a noetherian left $R$-module and a completely reducible left $R / J$-module for all positive integers $i$, the left $R$-module $J$ has a composition series, and is therefore artinian. Together with the minimum condition in $R / J$ this implies the minimum condition on left ideals in $R$.

$(8) \rightarrow(1)$ : Since $R / J$ is a direct sum of division rings, $T(R / J)=0$ by [21, Th. 5]. If $\varphi$ denotes the canonical epimorphism of $R$ onto $R / J$, then it follows from the radical property of $T$ that $(T(R)) \varphi \leqq$ $T(R \varphi)=T(R / J)=0$, whence $T(R)=J$. Since $R$ is left artinian, $N=J$, and $R$ has only finitely many prime ideals by 3.4 , so every prime ideal is corpoidal. Thus, every left $R$-module is admissible, and it follows from 2.7 that every indecomposable injective left $R$-module is of the form $E_{R}(R / P)$, where $P$ denotes some prime ideal of $R$. Since $N=N^{*}=J=T$, it follows from [15, Th. 2.3] that every injective indecomposable left $R$-module is isomorphic to a submodule of $E_{R}\left(R / N^{*}\right)$. Since a left noetherian ring is characterized by the property that its injective modules are direct sums of injective and indecomposable modules, this implies (1).

REMARK 3.7. In Theorem 3.6 the conditions (1) and (4) can be replaced by conditions $\left(1^{*}\right)$ and $\left(4^{*}\right)$ which originate from (1) and (4) if we replace $N^{*}$ by $T$. Because of $3.6(5)$ and $N^{*} \subseteq T$ it is clear that $\left(1^{*}\right)$ follows from $(1)$. Likewise $\left(4^{*}\right.$ b) and $\left(4^{*} \mathrm{c}\right)$ follow immediately from $\left(1^{*}\right)$ by means of [5, Th. 3.3]. Since the number of prime ideals of $R$ is less than or equal to the cardinality of $\pi(E(R / T))$ by $\left(1^{*}\right)$, it follows from 3.5 that every prime ideal is corpoidal, proving the minimum condition on left ideals in $R$ by 2.2. The proof from (3) to (4) in Theorem 3.6 shows that this implies $\left(4^{*} a\right)$. The proof from $\left(4^{*}\right)$ to (5) is nearly the same as the proof from (4) to (5) in Theorem 3.6, and Theorem 3.6 shows that (5) implies again (1).

The question arises whether in (1) and (4) of 3.6 the radical $N^{*}$ can also be replaced by smaller radicals like $J$ or $N$. The following 
simple example shows that this is not true in general. If $R$ is semisimple with minimum condition, the Jacobson radical $J$, the Koethe radical $K$, the Levitzky radical $L$ and the lower Baer nil radical $N$ are all equal to zero, and $E\left({ }_{R} R\right)={ }_{R} R$ is a cogenerator in the category of left $R$-modules, and $R$ satisfies also conditions (4a) and (4b) of 3.6. In general, however, $R$ is not a direct sum of division rings.

REMARK 3.8. Since $C(R / P)=S(R / P)$ for every prime ideal $P$ of a reduced left artinian ring $R$, and since every injective indecomposable left $R$-module is of the form $E_{R}(R / P)$ for some prime ideal $P$, the question arises, whether $S(E)=C(E)$ for every injective indecomposable left $R$-module $E$. According to [11,3.10] this, in turn, would imply $S(M)=C(M)$ for all left $R$-modules $M$. The following example shows that this is not true in general.

ExAmple 3.9. Let $Q$ be the field of rationals, and let $R$ be a $Q$-algebra whose generators $0, a, b, c, 1$ are multiplied according to the following table:

\begin{tabular}{c|ccccc} 
& 0 & $a$ & $b$ & $c$ & 1 \\
\hline 0 & 0 & 0 & 0 & 0 & 0 \\
$a$ & 0 & 0 & 0 & 0 & $a$ \\
$b$ & 0 & 0 & $b$ & $b$ & $b$ \\
$c$ & 0 & $a$ & $b$ & $c$ & $c$ \\
1 & 0 & $a$ & $b$ & $c$ & 1.
\end{tabular}

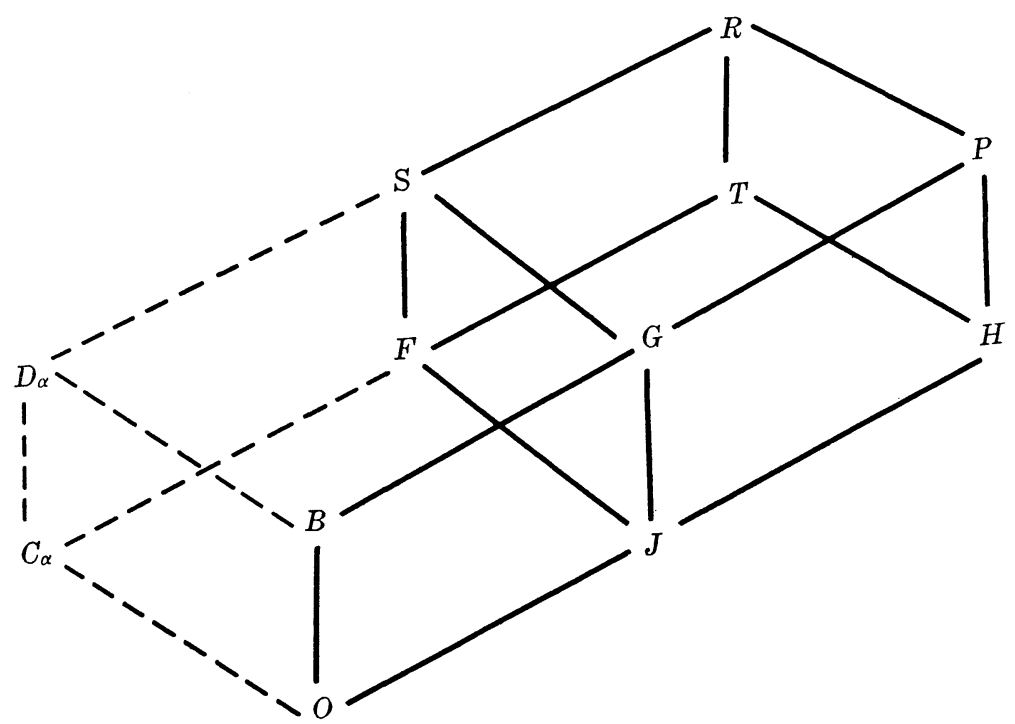


Then $R$ has the following left ideals (see [12, Example 9.2b]): $\{0\}$, $J=[a], B=[b], C_{\alpha}=[b-c+\alpha a]$ with $\alpha \in Q, D_{\alpha}=[b, c-\alpha a]$ with $\alpha \in Q, F=[a, b-c], G=[a, b], H=[a, c-1], S=[a, b, c], I=[a$, $b-c, c-1]$ and $P=[a, b, c-1]$, where $[x, y, z]$ denotes the left $Q$ vector space generated by the elements $x, y$ and $z$. The lattice of left ideals has the form shown in the diagram above. $R$ has an identity and is left artinian, the only left ideals that are not two-sided are the left ideals $C_{\alpha}$ and $D_{\alpha}$ for every $\alpha \in Q$. The prime ideals $S, T$ and $P$ are maximal left ideals. Since $P J=0$ and $J=R a$, it follows that $P=(R a)_{\ell}$, whence $P=a_{\ell}$, since $P$ is a maximal left ideal. Thus, $J=R a \simeq R a_{\ell}=$ $R / P$. Since $J$ is a large $R$-submodule of $H, E_{R}(J) \neq J$. If $0 \neq x \in J$, it follows that $x_{\ell}=P$, so $x_{\ell}$ is not large in $R$ since $P \cap C_{\alpha}=0$. Hence $Z_{R}(J)=0$, whence $C\left(E_{R}(J)\right)=E_{R}(J) \supset J=S(J)=S\left(E_{R}(J)\right)$ by [13, Propriété 3.4]. If, however, $R$ is a commutative artinian ring with identity, then $R$ is reduced, and furthermore $R$ has the property that heart and socle coincide for every unitary left $R$-module. Thus, the question arises, which additional conditions a noncommutative left artinian reduced ring must satisfy to permit the same conclusion. An answer to this problem is given in the following section.

\section{Finite direct sums of left artinian local rings.}

Proposition 4.1. Let $R$ be a left noetherian ring whose prime ideals are maximal, $E$ an injective indecomposable left $R$-module, $\operatorname{ch}(E)=\{P\}$. Then $P_{r}(E)=J_{r}(E)$.

Proof. As a maximal ideal $P$ is primitive, whence $R \supset P \supseteqq J \supseteqq 0$, which implies $0 \subset P_{r}(E) \subseteq J_{r}(E) \subseteq E$. By [19, Lemma 1], this implies $J_{r}(E) / P_{r}(E) \simeq_{R} \operatorname{Hom}_{R}(P / J, E)$. If $0 \neq \varphi \in \operatorname{Hom}_{R}(P / J, E)$, then it follows from $P_{r}(E) \leqq \subseteq^{\prime} E$ that $\operatorname{ch}\left((P / J) \emptyset \cap P_{r}(E)\right) \neq \varnothing$. Thus there exists a nonzero element $x \in(P / J) \varphi \cap P_{r}(E)$ with $P=(R x)_{\ell}$, whence $(P / J)_{\ell} \subseteq$ $\left((P / J)_{\ell}\right)_{\ell} \subseteq(R x)_{\ell}=P$. Since, by $3.4, R$ has only finitely many prime ideals $P, Q_{1}, \cdots, Q_{n}$, and since they are all primitive by assumption, it follows that

$$
\begin{aligned}
P / J & =P / P \cap Q_{1} \cap \cdots \cap Q_{n} \simeq\left(P+\left(Q_{1} \cap \cdots \cap Q_{n}\right)\right) / Q_{1} \cap \cdots \cap Q_{n} \\
& =R / Q_{1} \cap \cdots \cap Q_{n},
\end{aligned}
$$

whence $Q_{1} \cap \cdots \cap Q_{n}=(P / J)_{\ell} \subseteq P$, contradicting the maximality of the prime ideals of $R$. Thus, $\operatorname{Hom}_{R}(P / J, E)=0$, whence $J_{r}(E)=P_{r}(E)$.

THEOREM 4.2. The following properties of a ring $R$ are equivalent.

(1) $R$ is a direct sum of finitely many left artinian local rings.

(2) $\left\{\begin{array}{l}\text { (a) } R \text { is a reduced left artinian ring. } \\ \text { (b) } J P=P J \text { for every prime ideal } P \text { of } R \text {. }\end{array}\right.$ 
(3) $\{$ (a) $R$ is a reduced left artinian ring.

(b) $J P \subseteq P J$ for all prime ideals $P$ of $R$.

(4) $\{$ (a) $R$ is a reduced left artinian ring.

(b) $S(M)=C(M)$ for all left $R$-modules $M$.

(5) $\{$ (a) $R$ is left artinian.

(b) $L \cong$ ter $L$ for all left ideals $L$ of $R$.

(a) $R$ is left noetherian.

(6) $\{$ (b) $L \cong \operatorname{rad} L$ for all left ideals $L$ of $R$.

(c) Prime ideals of $R$ are maximal ideals.

Proof. (1) $\rightarrow(2)$ : If $R=\bigoplus_{i=1}^{n} R_{i}$ with left artinian local rings, and if $J_{i}$ denotes the Jacobson radical of $R_{i}$, then $J=\bigoplus_{i=1}^{n} J_{i}$. Obviously, the prime ideals of $R$ are the ideals of the form

$$
P_{i}=R_{1} \oplus \cdots \oplus R_{i-1} \oplus J_{i} \oplus R_{i+1} \oplus \cdots \oplus R_{n},
$$

so they are maximal left ideals, since $R_{i} / J_{i}$ is a division ring. Thus, $R$ is a reduced left artinian ring by Theorem 3.6. Since every $R_{i}$ has an identity, it follows that for every prime ideal $P$ of $R$

$$
\begin{aligned}
P J & =R_{1} J_{1} \oplus \cdots \oplus R_{i-1} J_{i-1} \oplus J_{i}^{2} \oplus R_{i+1} J_{i+1} \oplus \cdots \oplus R_{n} J_{n} \\
& =J_{1} \oplus \cdots \oplus J_{i-1} \oplus J_{i}^{2} \oplus J_{i+1} \oplus \cdots \oplus J_{n} \\
& =J_{1} R_{1} \oplus \cdots \oplus J_{i-1} R_{i-1} \oplus J_{i}^{2} \oplus J_{i+1} R_{i+1} \oplus \cdots \oplus J_{n} R_{n}=J P .
\end{aligned}
$$

Trivially, (3) follows from (2).

$(3) \rightarrow(4)$ : Assume $E$ is an injective indecomposable left $R$-module with $S(E) \neq C(E)$. If $x$ is a nonzero element in $C(E)$ such that the submodule $R \bar{x}$ of $C(E) / S(E)$ generated by $\bar{x}=x+S(E)$ is minimal, then $Q=\bar{x}_{\ell}$ is a prime ideal by Theorem 3.6. By Theorem 2.7, $E \simeq E_{R}(R / P)$ for some prime ideal $P$. By [13, Th. 3.1], $x_{\ell}$ is maximal in the set $\left\{e_{\ell} \mid 0 \neq e \in E\right\}$. The admissibility of $E$ implies that $P$ is contained in this set, whence $x_{\ell} \nsubseteq P$, since otherwise $x_{\ell}=P$ and thus $x \in P_{r}(E)=S(E)$. Since $R$ has an identity, $x_{\ell}$ is contained in some maximal left ideal $Q_{1} \neq P$ of $R$, which is a prime ideal by 3.6. From $Q x \subseteq S(E)=P_{r}(E)$ it follows that $P Q \subseteq x_{\ell} \subseteq Q_{1}$, whence $Q=Q_{1}$. Since $P J x \subseteq P Q x=0$, (3b) implies that also $J P x=0$, whence $P x \leqq$ $J_{r}(E)=P_{r}(E)$ by 4.1. Thus, $P^{2} \cong Q_{1}$, a contradiction. Hence $S(E)=$ $C(E)$ for every injective indecomposable module $R$, which implies (4b) by $[11,3.10]$.

$(4) \rightarrow(5)$ : If $L$ is an irreducible left ideal, then $L$ is tertiary, and ter $L=P$ is a prime ideal. If $K$ is maximal in the set of $P$ tertiary left ideals containing $L$, then $K$ is irreducible by [12, Th. 8.3]. Assume that there exists a left ideal $M$ of $R$ with $K \subset M \subset R$ and $\pi(R / M)=\pi(R / K)$, then the left $R$-module $R / M$ is homogeneous because of $|\pi(R / K)|=1$. By [12, Propriété 10.11], this implies that 
$M$ is tertiary and hence $P$-tertiary because of $\operatorname{ch}(R / M)=\operatorname{ch}(R / K)$, contradicting the choice of $K$. Thus, $S(R / K)=C(R / K)=R / K$ by (4b) and [11, Satz 3.1], implying that $K$ is a maximal left ideal and hence a prime ideal by Theorem 3.6. Therefore $\{K\}=\operatorname{ch}(R / K)=$ \{ter $K\}=\{P\}$, whence $L \subseteq K=P=\operatorname{ter} L$. If $L$ is any left ideal of $R$ and if $L=\bigcap_{i=1}^{n} L_{i}$ with irreducible left ideals $L_{i}$, then it follows from [12, Propriété 7.7] and the preceding argument that

$$
L=\bigcap_{i=1}^{n} L_{i} \subseteq \bigcap_{i=1}^{n} \operatorname{ter} L_{i} \subseteq \operatorname{ter}\left(\bigcap_{i=1}^{n} L_{i}\right)=\operatorname{ter} L .
$$

$(5) \rightarrow(6)$ : It is clear that (6a) and (6c) follow from (5a). Assume that the set $\phi$ of all left ideals $L$ of $R$ with $L \nsubseteq \operatorname{rad} L$ is nonempty, and let $K$ be maximal in $\phi$ and $M$ minimal in the set of all left ideals of $R$ which contain $K$ properly. Then $K$ is an irreducible left ideal, since $K=K_{1} \cap K_{2}$ with $K_{1} \supset K$ and $K_{2} \supset K$ would imply $K=K_{1} \cap K_{2} \subseteq$ $\operatorname{rad} K_{1} \cap \operatorname{rad} K_{2}=\operatorname{rad} K$. Thus, $K$ is tertiary and $\operatorname{ch}(R / K)=\{\operatorname{ter} K\}$. Since $M / K$ is a minimal submodule of $R / K$, it follows that $(M / K)_{\ell}=$ ter $K$. Since $\operatorname{rad} M$ is nil modulo $(R / M)_{\ell}$, and since $R$ is left noetherian, there exists a positive integer $n$ such that $(\operatorname{rad} M)^{n} \subseteq(R / M)_{\ell}$, whence ter $K \cdot(\operatorname{rad} M)^{n} R \subseteq K$ and thus ter $K \cdot(\operatorname{rad} M)^{n} \subseteq(R / K)_{\ell} \subseteq \operatorname{rad} K$. If $\left\{Q_{1}, \cdots, Q_{m}\right\}$ is the set of prime ideals different from ter $K$ containing $(R / K)_{\ell}$, then $\operatorname{rad} M \subseteq Q_{i}$ for $i=1, \cdots, m$. Since $M \subseteq \operatorname{rad} M$, this implies

$$
\begin{aligned}
K & =K \cap \text { ter } K \subseteq M \cap \text { ter } K \leqq \operatorname{rad} M \cap \text { ter } K \\
& \leqq Q_{1} \cap \cdots \cap Q_{m} \cap \text { ter } K=\operatorname{rad} K
\end{aligned}
$$

a contradiction. Thus, $\phi$ is empty, and $(6 \mathrm{~b})$ is true.

$(6) \rightarrow(1)$ : Since $\operatorname{rad} L \subseteq$ ter $L$, it follows that $L \cong$ ter $L$ for every left ideal $L$. If $L$ is a maximal left ideal, then $L=\operatorname{ter} L$, and since maximal left ideals are tertiary, $L$ is a prime ideal. Together with (6b) this implies that prime ideals are maximal left ideals, so $R$ is reduced and left artinian by Theorem 3.6. Assume that the set $\phi$ of all left ideals $L$ with $\operatorname{rad} L \neq \operatorname{ter} L$ is nonempty, and let $K$ be maximal in $\dot{\phi} . \quad K$ is an irreducible left ideal since otherwise $K=\bigcap_{i=1}^{n} K_{i}$ with $P_{i}$-tertiary left ideals $K_{i}$, where none of the $K_{i}$ is superfluous and $P_{i} \neq P_{j}$ for $i \neq j$. But then, by [12, Th. 8.3] and [12, Corollaire 8.4], ter $K=\bigcap_{i=1}^{n}$ ter $K_{i}=\bigcap_{i=1}^{n} \operatorname{rad} K_{i}=\operatorname{rad}\left(\bigcap_{i=1}^{n} K_{i}\right)=\operatorname{rad} K$, a contradiction. If $N$ is minimal in the set of left ideals which contain $K$ properly, then either $K=N \cap \operatorname{rad} K$ or $N=N \cap \operatorname{rad} K$, since $K \subseteq$ $\operatorname{rad} K$. The first case yields $K=\operatorname{rad} K$ by the irreducibility of $K$, so $\operatorname{rad} K$ is a prime ideal. Since $\operatorname{rad} K \subseteq$ ter $K$ and prime ideals are maximal, this yields a contradiction. Thus, $N=N \cap \operatorname{rad} K \cong \operatorname{rad} K$, whence $\operatorname{rad} N \subseteq \operatorname{rad} \operatorname{rad} K=\operatorname{rad} K$. Since $K \subset N$ implies $\operatorname{rad} K \subseteq \operatorname{rad} N$, 
this $\operatorname{implies} \operatorname{rad} K=\operatorname{rad} N=\operatorname{ter} N \subseteq$ ter $K$ by the maximality of $K$ in $\phi$.

If $E$ is an injective and indecomposable left $R$-module and if $x$ is a nonzero element of $C(E)$, then $C(R x)=R x \cap C(E(R x))=R x \cap C(E)=$ $R x$, so there exists no submodule $N \subset R x$ with $\pi(R x / N)=\pi(R x)$ by [11, Satz 3.1]. Since every left $R$-module is admissible, it follows from [11, Folgerung 2.8] that there exists no left ideal $L$ of $R$ with $x_{\ell} \subset L \subset R$ and $\operatorname{ch}(R / L)=\operatorname{ch}\left(R / x_{\ell}\right)$, whence no ter $x_{\ell}$-tertiary left ideal contains $x_{\ell}$ properly. Thus $x_{\ell}$ is a maximal ter $x_{\ell}$-tertiary left ideal, whence $x_{\ell}=\operatorname{ter} x_{\ell}$. Thus, $x_{\ell}$ is a maximal left ideal, which implies $x \in S(E)$ and thus $S(E)=C(E)$. By [11, 3.10], this implies $S(M)=C(M)$ for every module $M$. Consequently, in particular $C(R / K)=S(R / K)=N / K$, where the second equation follows from the irreducibility of $K$ and the choice of $N$. By [11, Satz 3.1], $C(R / K)$ is the intersection of all submodules $0 \neq M / K \cong R / K$ with $\pi(R / M)=\pi(R / K)$. Since $R$ is left artinian, $C(R / K)=N / K$ is already the intersection of finitely many of these submodules, whence $\pi(R / N)=$ $\pi(R / K)$ by [12, Propriété 10.13], so that $\operatorname{ch}(R / N)=\operatorname{ch}(R / K)=$ \{ter $K\}$. Since ter $N$ is the intersection of all prime ideals associated with the left $R$-module $R / N$, it follows that ter $N=\operatorname{ter} K$, so that $\operatorname{rad} K=$ ter $K$ by the equation in the previous paragraph, a contradiction to the choice of $K$. Thus, ter $L=\operatorname{rad} L$ for all left ideals $L$. Therefore tertiary left ideals are primary, and since prime ideals of $R$ are maximal left ideals, this implies (1) by [11, Satz 4.2]. Since a commutative artinian ring is a direct sum of local rings, we get:

COROLlary 4.3. A commutative noetherian ring $R$ is artinian if and only if $S(M)=C(M)$ for every left $R$-module $M$.

Furthermore, the last part of the theorem shows that the following is true:

COROLlary 4.4. If $R$ is a left noetherian ring whose left $R$ modules are admissible and have coinciding heart and socle, then $R$ has the Artin-Rees-property for left ideals.

In fact, Theorem 4.2 is an extension of Satz 4.2 in [11]. Thus, if a ring $R$ has properties (1)-(6) of Theorem 4.2, ch $(M)=\operatorname{supp}(M)$ for every left $R$-module $M$, which gives a partial answer to the question raised in 2.6 .

It would be interesting to know, whether the inclusion in condition (3) of 4.2 can be replaced by the reverse inclusion. Considering the manner how this inclusion was used in the proof of 4.2 , this does 
not seem to be true, we have, however, not been able to find a counter-example.

\section{REFERENCES}

1. H. Bass, Finitistic dimension and a homological generalization of semi-primary rings, Trans. Amer. Math. Soc. 95 (1960), 466-488.

2. R. Brauer, Some remarks on associative rings and algebras, Nat. Acad. Sci.-Nat. Research Council Publication 502 (1957), 4-11.

3. S. U. Chase, Direct products of modules, Trans. Amer. Math. Soc. 97 (1960), 457-473.

4. R. Croisot, Séminaire P. Dubreil et C. Pisot 1956/57, Algèbre et Théorie des Nombres, Exposé 22.

5. C. Faith, and E. A. Walker, Direct-sum representations of injective modules, J. Algebra 5 (1967), 203-221.

6. A. I. Gerčikov, Rings, decomposable into the direct sum of fields, Mat. Sb. (N. S.) (49) 7 (1940), 591-597.

7. A. W. Goldie, Semi-prime rings with maximum condition, Proc. London Math. Soc.

(3) 10 (1960), 201-220.

8. - Rings with maximum condition, Lecture Notes, Yale University, 1961.

9. R. Gordon, Rings faithfully represented on their left socle, J. Algebra 7 (1967), 303-342.

10. K. Kishimoto and Y. Kurata, Note on decomposition sets of semi-prime rings, J. Fac. Sc. Hokkaido Univ. 16 (1962), 214-220.

11. G. Krause, Moduln ïber noetherschen Ringen, ihr Sockel and ihr Herz, ihre Charakteristik und ihr Träger, J. Reine Angew. Math., 235 (1969), 133-145.

12. L. Lesieur and R. Croisot, Algèbre noetherienne non-commutative, Mém. des. Sc. Math. 154 (1963).

13. - Coeur d'un module, J. de Math. Pures et Appl. 42 (1963), 367-406.

14. L. Levy, Unique subdirect sums of prime rings, Trans. Amer. Math. Soc. 106 (1963), 64-76.

15. E. Matlis, Injective modules over noetherian rings, Pacific J. Math. 8 (1958), 511-528.

16. G. Michler, Kleine Ideale, Radikale und die Eins in Ringen, Publ. Math. Debrecen 12 (1965), 231-252.

17. —, Radikale und Sockel, Math. Ann. 167 (1966), 1-48.

18. B. L. Osofsky, A generalization of quasi-Frobenius rings, J. Algebra 4 (1966), 373-387.

19. A. Rosenberg and D. Zelinsky, Finiteness of the injective hull, Math. Z. 70 (1959), 372-380.

20. F. Szász, Über Ringe mit Minimalbedingung für Hauptrechtsideale I, Publ. Math. Debrecen 7 (1960), 54-64.

21. G. Thierrin, Sur le radical corpoidal d'un anneau, Canad. J. Math. 12 (1960), 101-106.

Received December 23, 1968.

UNIVERSITY OF MANITOBA

Washington State University, Pullman 



\section{PACIFIC JOURNAL OF MATHEMATICS}

\section{EDITORS}

H. SAMelson

Stanford University

Stanford, California 94305

\section{Richard PIERCE}

University of Washington

Seattle, Washington 98105
J. DugundjI

Department of Mathematics

University of Southern California

Los Angeles, California 90007

BASIL GoRDON*

University of California

Los Angeles, California 90024

\section{ASSOCIATE EDITORS}
E. F. BECKENBACH
B. H. NeumanN
F. WOLE
K. YosHIDA

\section{SUPPORTING INSTITUTIONS}

UNIVERSITY OF BRITISH COLUMBIA

CALIFORNIA INSTITUTE OF TECHNOLOGY

UNIVERSITY OF CALIFORNIA

MONTANA STATE UNIVERSITY

UNIVERSITY OF NEVADA

NEW MEXICO STATE UNIVERSITY

OREGON STATE UNIVERSITY

UNIVERSITY OF OREGON

OSAKA UNIVERSITY

UNIVERSITY OF SOUTHERN CALIFORNIA

\author{
STANFORD UNIVERSITY \\ UNIVERSITY OF TOKYO \\ UNIVERSITY OF UTAH \\ WASHINGTON STATE UNIVERSITY \\ UNIVERSITY OF WASHINGTON \\ * * * * \\ AMERICAN MATHEMATICAL SOCIETY \\ CHEVRON RESEARCH CORPORATION \\ TRW SYSTEMS \\ NAVAL WEAPONS CENTER
}

The Supporting Institutions listed above contribute to the cost of publication of this Journal, but they are not owners or publishers and have no responsibility for its content or policies.

Mathematical papers intended for publication in the Pacific Journal of Mathematics should be in typed form or offset-reproduced, (not dittoed), double spaced with large margins. Underline Greek letters in red, German in green, and script in blue. The first paragraph or two must be capable of being used separately as a synopsis of the entire paper. The editorial "we" must not be used in the synopsis, and items of the bibliography should not be cited there unless absolutely necessary, in which case they must be identified by author and Journal, rather than by item number. Manuscripts, in duplicate if possible, may be sent to any one of the four editors. Please classify according to the scheme of Math. Rev. 36, 1539-1546. All other communications to the editors should be addressed to the managing editor, Richard Arens, University of California, Los Angeles, California, 90024.

50 reprints are provided free for each article; additional copies may be obtained at cost in multiples of 50 .

The Pacific Journal of Mathematics is published monthly. Effective with Volume 16 the price per volume (3 numbers) is $\$ 8.00$; single issues, $\$ 3.00$. Special price for current issues to individual faculty members of supporting institutions and to individual members of the American Mathematical Society: $\$ 4.00$ per volume; single issues $\$ 1.50$. Back numbers are available.

Subscriptions, orders for back numbers, and changes of address should be sent to Pacific Journal of Mathematics, 103 Highland Boulevard, Berkeley, California, 94708.

PUBLISHED BY PACIFIC JOURNAL OF MATHEMATICS, A NON-PROFIT CORPORATION

Printed at Kokusai Bunken Insatsusha (International Academic Printing Co., Ltd.), 7-17, Fujimi 2-chome, Chiyoda-ku, Tokyo, Japan.

* Acting Managing Editor. 


\section{Pacific Journal of Mathematics}

\section{Vol. 33, No. $2 \quad$ April, 1970}

Raymond Balbes and Alfred Horn, Projective distributive lattices ....... 273

John Findley Berglund, On extending almost periodic functions ......... 281

Günter Krause, Admissible modules and a characterization of reduced left

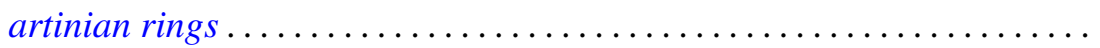

Edward Milton Landesman and Alan Cecil Lazer, Linear eigenvalues and a nonlinear boundary value problem ....................... 311

Anthony To-Ming Lau, Extremely amenable algebras ............... 329

Aldo Joram Lazar, Sections and subsets of simplexes .............. 337

Vincent Mancuso, Mesocompactness and related properties ............. 345

Edwin Leroy Marsden, Jr., The commutator and solvability in a generalized orthomodular lattice .................................. 357

Shozo Matsuura, Bergman kernel functions and the three types of canonical domains.......................................... 363

S. Mukhoti, Theorems on Cesàro summability of series .............. 385

Ngô Van Quê, Classes de Chern et théorème de Gauss-Bonnet ........... 393

Ralph Tyrrell Rockafellar, Generalized Hamiltonian equations for convex problems of Lagrange ................................ 411

Ken iti Sato, On dispersive operators in Banach lattices ............. 429

Charles Andrew Swanson, Comparison theorems for elliptic differential

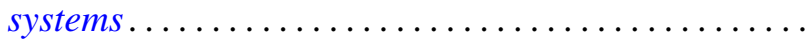

John Griggs Thompson, Nonsolvable finite groups all of whose local subgroups are solvable. II

David J. Winter, Cartan subalgebras of a Lie algebra and its ideals ... 